\title{
Medical Journalism at the Time of the COVID-19 Pandemic
}

\author{
Rochita Venkataramanan ${ }^{1}$ Argha Chatterjee ${ }^{2}$ \\ ${ }^{1}$ Department of Clinical Radiology, Apollo Hospital, Chennai, India \\ ${ }^{2}$ Department of Radiology, Tata Medical Centre, Kolkata, India
}

J Gastrointestinal Abdominal Radiol ISGAR:2020;3:115-115

An unprecedented outbreak of pneumonia of unknown etiology in Wuhan city, Hubei province, in China emerged in December 2019. A novel Coronavirus was identified as the causative agent, and the disease was subsequently termed COVID-19 by the World Health Organization. The COVID-19 pandemic has rapidly spread through the continents; also, it has brought along with it great hardships on the medical fraternity, as they cope with the rising numbers of sick. What makes these times especially challenging is that there is no clear understanding of which medications or treatment protocols are effective, as this disease is leaving the treating doctors groping in the dark. At the root of this dilemma is the quality of medical literature that is coming forth with studies showing clear flaws in methodology. There are currently 19251 articles indexed in PubMed on the topic of COVID published within a span of 5 months. ${ }^{1}$ It is understandable that such volume of articles is not undergoing a rigorous peer review process at such a short time. What further muddied the waters is that two of the most prestigious medical journals having published studies relating to COVID-19 treatment based on big data, retracted them after their accuracy was questioned by researchers across the world. ${ }^{2}$

Such an event has dented the faith that the medical fraternity has in medical journals, a crushing blow leaving us gasping for breath and wondering how this could have happened. Publishing inaccurate research is a form of misconduct that is well known and may have several reasons. It has been known that even prestigious journals publish trials that are funded by the industry, the results of which are rarely unfavorable to the companies. ${ }^{3}$ Journals are known to pursue publicity and would like to publish sensational articles with groundbreaking revelations. Authors may have conflicting interests and yet these may be hard to identify if not honestly disclosed.
Peer review, a process which should be able to detect fallacies in research, is itself not a robust tool. It is known to be ineffective at finding fraud and prone to bias and abuse. Ill practices in which authors have been caught reviewing their own papers or suggested their friends as reviewers are known. ${ }^{4}$

During this time of a pandemic, medical journalism should provide answers to save lives and act as beacons of hope. Researchers should also make sure that they do not submit inaccurate data or use questionable research practices, misdirecting medical treatment. If scientific research is false, it will shake the very foundation of the pillars on which mankind survives. Falsehood in scientific research needs to be tackled by a united honest front of editors, reviewers and authors for the good of mankind.

\section{Conflict of Interest}

None declared.

\section{References}

1 Chen Q, Allot A, Lu Z. Keep up with the latest coronavirus research. Nature 2020;579(7798):193

2 Mehra MR, DesaiSS, Ruschitzka F, Patel AN. Hydroxychloroquine or chloroquine with or without a macrolide for treatment of COVID-19: a multinational registry analysis. Lancet 2020; (e-pub ahead of print). doi: 10.1016/ S0140-6736(20)31324-6

3 Smith R. The trouble with medical journals. J R Soc Med 2006;99(3):115-119

4 Horbach SPJM, Halffman W. The ability of different peer review procedures to flag problematic publications. Scientometrics 2019;118(1):339-373
Address for correspondence

Rochita Venkataramanan, MD, DNB, DMRD, Department of Clinical

Radiology, Apollo Hospital, Chennai, India (e-mail: rochitav@yahoo.com; rochitav@gmail.com).
DOI https://doi.org/ $10.1055 / \mathrm{s}-0040-1714180$ ISSN 2581-9933.
(C)2020 by Indian Society of

Gastrointestinal and Abdominal Radiology
License terms

() (1) $\Theta \circledast$ 